\title{
Evaluation of condyle defects using different reconstruction protocols of cone-beam computed tomography
}

\author{
Luana Costa Bastos(a) \\ Paulo Sérgio Flores Campos ${ }^{(a)}$ \\ Flávia Maria de Moraes \\ Ramos-Perez ${ }^{(\mathbf{b})}$ \\ Andrea dos Anjos Pontual(c) \\ Solange Maria Almeida(d)
}

(a) Department of Oral and Maxillofacial Radiology, School of Dentistry, Universidade Federal da Bahia - UFBA, Salvador, BA, Brazil.

(b) Departament of Clinical and Preventive Dentistry, School of Dentistry, Universidade Federal de Pernambuco - UFPE, Recife, PE, Brazil.

(c) Department of Oral Radiology, School of Dentistry, Universidade Federal de Pernambuco - UFPE, Camaragibe, PE, Brazil.

(d) Department of Oral Radiology, School of Dentistry of Piracicaba, Universidade Estadual de Campinas - Unicamp, Piracicaba, SP, Brazil.

Declaration of Interests: The authors certify that they have no commercial or associative interest that represents a conflict of interest in connection with the manuscript.

Corresponding Author:

Luana Costa Bastos

E-mail: bastosluana@ymail.com

htrp://dx.doi.org/10.1590/S1806-83242013000600010

Submitted: Apr 03, 2013

Accepted for publication: Jul 31, 2013

Last revision: Aug 17, 2013
Abstract: This study was conducted to investigate how well cone-beam computed tomography (CBCT) can detect simulated cavitary defects in condyles, and to test the influence of the reconstruction protocols. Defects were created with spherical diamond burs (numbers 1013, 1016, 3017 ) in superior and/or posterior surfaces of twenty condyles. The condyles were scanned, and cross-sectional reconstructions were performed with nine different protocols, based on slice thickness $(0.2,0.6,1.0 \mathrm{~mm})$ and on the filters (original image, Sharpen Mild, S9) used. Two observers evaluated the defects, determining their presence and location. Statistical analysis was carried out using simple Kappa coefficient and $\mathrm{McNe}-$ mar's test to check inter- and intra-rater reliability. The chi-square test was used to compare the rater accuracy. Analysis of variance (Tukey's test) assessed the effect of the protocols used. Kappa values for inter- and intra-rater reliability demonstrate almost perfect agreement. The proportion of correct answers was significantly higher than that of errors for cavitary defects on both condyle surfaces $(p<0.01)$. Only in identifying the defects located on the posterior surface was it possible to observe the influence of the $1.0 \mathrm{~mm}$ protocol thickness and no filter, which showed a significantly lower value. Based on the results of the current study, the technique used was valid for identifying the existence of cavities in the condyle surface. However, the protocol of a $1.0 \mathrm{~mm}$-thick slice and no filter proved to be the worst method for identifying the defects on the posterior surface.

Descriptors: Mandibular Condyle; Bone Diseases; Cone-Beam Computed Tomography; Image Enhancement.

\section{Introduction}

Most pathological conditions affecting the temporomandibular joint (TMJ) cause changes that begin in the covering soft tissue and may progress to involve the bones. In the case of the condyles, erosions-ranging from minor injuries (cavitary defects) to the complete absence of condyle regions- are one of the most common degenerative changes observed in radiographs, second only to flattening. ${ }^{1-3}$

Cavitary defects represent the initial stage of degenerative changes, indicating that TMJ is unstable, and that changes in bone surfaces are occurring. Radiographically, TMJ is described as a focal area with reduced density of the cortical and/or subjacent medullary bones. Erosions 
can develop, compromising condylar structures, reducing joint space, causing anterior open bite and, in some cases, ankylosis. ${ }^{4}$

Imaging exams are extremely important for early detection of erosions, since these may be the first local manifestation of certain serious systemic conditions (i.e. rheumatoid arthritis). The earlier the diagnosis and implementation of an appropriate treatment plan, the more favorable the patient's prognosis, by obviating major sequelae and ensuring quality of life. ${ }^{4}$ Minimal overlapping joint images are required to detect these lesions precisely. Some studies conclude in favor of conventional tomography, whereas others recommend multislice computed tomography (MSCT), but there is still no agreement about what technique should be established as the gold standard..$^{5-7}$

Cone beam computed tomography (CBCT), a technology based on the volumetric rendering of an area of interest, has been used in many countries to evaluate these changes. Similar to the MSCT, the CBCT offers post-processing possibilities for the images in which some parameters may be changed (i.e., slice thickness, filter application, etc.) to improve image quality without having to expose the patient again. ${ }^{6}$

Slice thickness may vary greatly depending on the equipment and the commercially available software. $^{7}$ The correct choice of what thickness should be used must take into account the evaluated anatomical structure and the suspected diagnosis. ${ }^{8}$

The influence of filters in reducing the noise of digital radiographic images has been studied extensively in regard to ability of the filters to detect interproximal bone loss, carious lesions and root fractures. $^{9-11}$ Filters are computational resources that work through algorithmic operations, determining changes in the image characteristics (i.e., enhancement or smoothing of the grayscale). ${ }^{11,12}$

There is a lack of studies in the literature on the combined influence of slice thickness and filters on CBCT exams. Therefore, the aim of this study was to compare the ability of different reconstruction protocols (varying thicknesses and types of filter) of CBCT images in detecting artificially produced cavitary bone defects in the mandibular condyle.

\section{Methodology}

This prospective study was reviewed and approved by the Research Ethics Committee of the School of Dentistry of Piracicaba, Universidade Estadual de Campinas - Unicamp (\# 169/2009). CBCT scans of ten dry human mandibles (not discriminated by gender and with an average age estimated at 40 years) with twenty sound condyles (without cavitary defects of any kind) were included in this study. Twenty defects were randomly produced in superior (10) and posterior (10) surfaces of the condyles, involving only cortical or both the cortical and the medullary bone, for the purpose of simulating the various stages of degenerative joint disease. ${ }^{13}$ The defects were produced using three different sizes of spherical diamond burs (KG Sorensen, Cotia, Brazil) mounted in a high-speed dental handpiece. The lesions produced had approximately the diameter of the burs used $(1.2 \mathrm{~mm}, 1.8 \mathrm{~mm}$ and $2.5 \mathrm{~mm}$, respectively) and the depth of each was equal to half of its diameter $(0.6 \mathrm{~mm}, 0.9 \mathrm{~mm}$ and $1.25 \mathrm{~mm}$, respectively). In each condyle, either one or two defects of different or the same diameter were made, or else no defect at all was introduced (Figure 1).

The mandibles were immersed in a Styrofoam box filled with water and positioned in the cephalostat of the i-CAT ${ }^{\circledR}$ CBCT scanner (Imaging Sciences International, Hatfield, USA) simulating an in vivo position (i.e., slightly angled on a horizontal plane), using the following acquisition protocol: $120 \mathrm{kV}$,

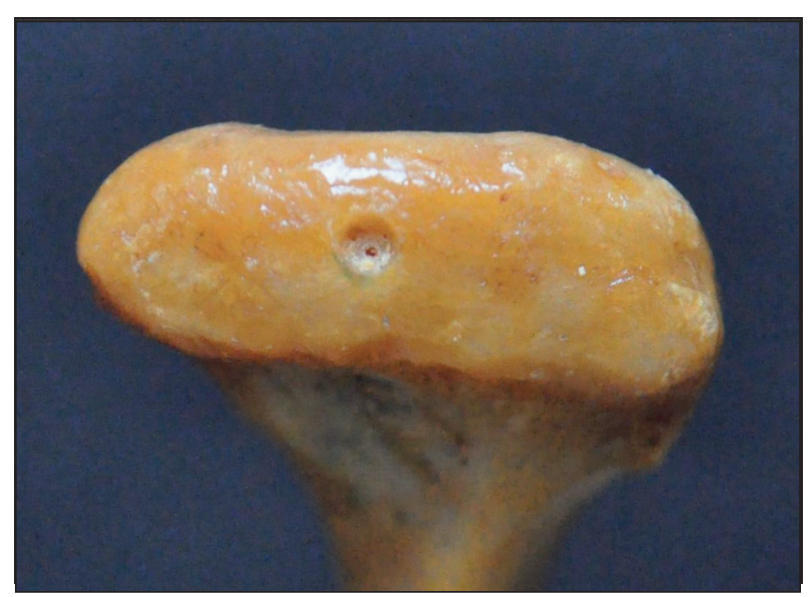

Figure 1 - Example of a medium defect created in the superior surface of the condyle. 

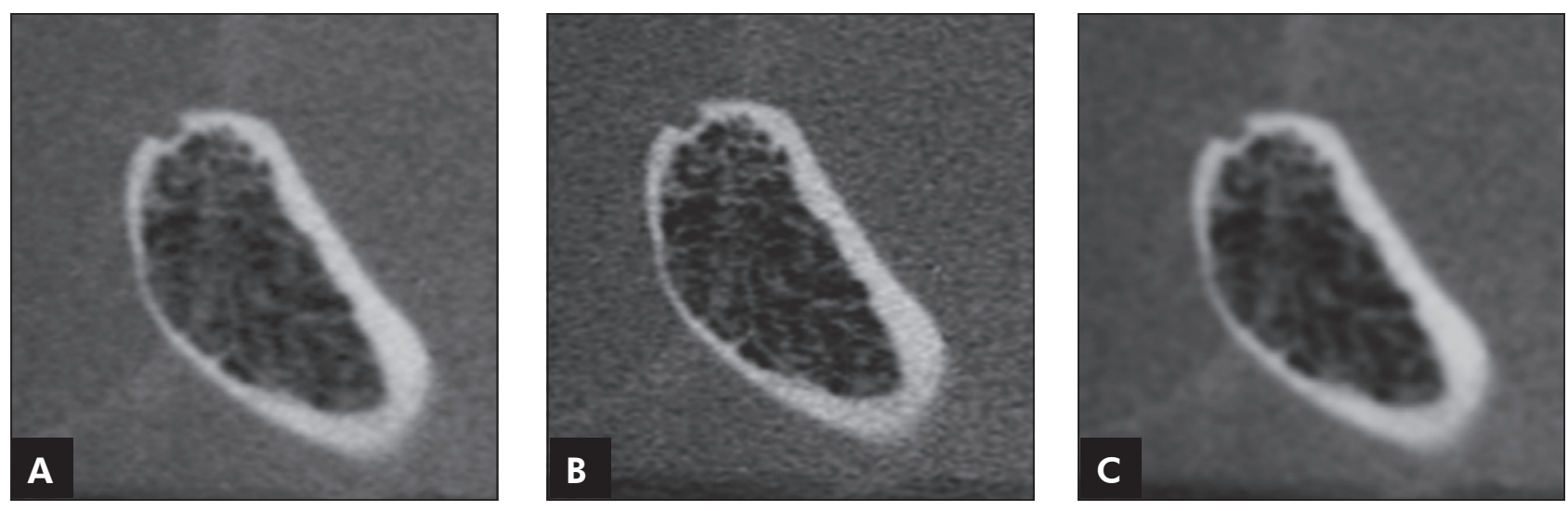

Figure 2 - Cross-sectional slices of the condyle in Figure 1 reconstructed in protocols 1, 2 and 3: (A) 0.2 mm-thick section and no filter; (B) $0.2 \mathrm{~mm}$-thick section and Sharpen Mild filter; (C) $0.2 \mathrm{~mm}$-thick section and S9 filter.

$36.12 \mathrm{mAs}$, field of view of $6 \mathrm{~cm}, 512 \times 512$ matrix, voxel size of $0.25 \mathrm{~mm}$ and acquisition time of $40 \mathrm{~s}$.

An independent workstation running Xoran$\mathrm{Cat}^{\mathrm{TM}}$ software (version 3.1.62, Xoran Technologies, Ann Arbor, USA) was used to select the multiplanar reconstruction (MPR) display mode, according to the following procedure: based on an axial image, the reconstructions were set perpendicular to the long axis of the condyle to generate the cross-sectional images. The following slice thicknesses were then selected for the cross-sectional reconstructions:

- thin $(0.2 \mathrm{~mm})$,

- medium (0.6 mm), or

- thick $(1.0 \mathrm{~mm})$.

The cross-sectional slices were saved in XSTD format and blindly evaluated individually by two oral and maxillofacial radiologists, with over five years of CBCT experience. Evaluation was conducted under dim light, in a workstation with a 22" flat-screen monitor (Dell Precision 390 ${ }^{\mathrm{TM}}$, Dell Inc., Round Rock, USA) using XoranCat ${ }^{\mathrm{TM}}$ software, which allows manipulating brightness and contrast, following nine different reconstruction protocols (Figure 2). Two filters that work distinctly were selected. The Sharpen Mild filter (an enhancement filter) intensifies density level differentiation (gray tones). This makes the boundaries more evident and the bone structure images clearer to see, ultimately allowing better assessment in certain cases. The $S 9$ filter (a smoothing filter) acts uniformly, calculating an average value for a group of pixels, and redistributing the density mean among the pixels, resulting in an image that has more uniform gray tones and is less granular.

The interpretation was carried out in two sessions with a fifteen day interval, and the images were presented randomly. The observers had to detect and locate (on superior or posterior surfaces) the bone defects. The gold standard was the macroscopic inspection of condyle defects.

The statistical analysis was carried out using SAS software (release 9.2 - TS Level 2M0; SAS Institute Inc., Cary, USA). The following tests were applied:

- Kappa coefficients and McNemar's test (to check inter- and intra-rater reliability),

- chi-square test (to compare the accuracy rates) and

- Tukey's test (analysis of variance, to contemplate the effect of the protocols used), with a significance level of $5 \%(p=0.05)$.

\section{Results}

Inter-rater reliability showed values greater than 0.8654 , which indicate almost perfect agreement between the observers or raters, in both the first and second session. Additionally, there was no significant difference between the observations (Table 1).

Regarding the intra-rater reliability, when comparing the responses given by Observer 2 for the identification of defects on the posterior surface, there was a complete absence of discordant pairs, 
Table 1 - Inter-rater reliability results.

\begin{tabular}{c|c|c|c|c|c|c}
\hline \multirow{2}{*}{ Session } & \multirow{2}{*}{$\begin{array}{c}\text { Defect } \\
\text { surface }\end{array}$} & \multicolumn{2}{|c|}{ McNemar's test } & \multicolumn{3}{c}{ Simple Kappa coefficient } \\
\cline { 3 - 7 } & Statistic & $p$-value & Kappa & \multicolumn{2}{|c}{ Confidence interval (95\%) } \\
\hline 1 & Superior & 3.5714 & 0.0588 & 0.9218 & 0.8650 & 0.9785 \\
\hline 1 & Posterior & 3.000 & 0.0833 & 0.8654 & 0.7920 & 0.9388 \\
\hline 2 & Superior & 2.6667 & 0.1025 & 0.9333 & 0.8809 & 0.9857 \\
\hline 2 & Posterior & 1.3333 & 0.2482 & 0.8661 & 0.7930 & 0.9392 \\
\hline
\end{tabular}

Table 2 - Intra-rater reliability results.

\begin{tabular}{c|c|c|c|c|c|c}
\hline \multirow{2}{*}{ Observer } & \multirow{2}{*}{$\begin{array}{c}\text { Defect } \\
\text { surface }\end{array}$} & \multicolumn{2}{|c|}{ McNemar's test } & \multicolumn{3}{|c}{ Simple Kappa coefficient } \\
\cline { 3 - 7 } & Statistic & $p$-value & Kappa & Confidence interval (95\%) \\
\hline 1 & Superior & 0.0000 & 1.0000 & 0.9777 & 0.9469 & 1.0000 \\
\hline 1 & Posterior & 1.0000 & 0.3173 & 0.9549 & 0.9112 & 0.9986 \\
\hline 2 & Superior & 0.3333 & 0.5637 & 0.9665 & 0.9290 & 1.0000 \\
\hline 2 & Posterior & - & - & 1.0000 & 1.0000 & 1.0000 \\
\hline
\end{tabular}

Table 3 - Frequency and percentage of correct observations in determining the defects in the images, and chi-square test for equality of proportions.

\begin{tabular}{c|c|c|c|c}
\hline Surface & Observation & Frequency & Percentage & $p$-value \\
\hline \multirow{2}{*}{ Superior } & Incorrect & 13 & 1.81 & \multirow{2}{*}{$<0.0001^{*}$} \\
\cline { 2 - 4 } & Correct & 706 & 98.19 & \\
\hline \multirow{2}{*}{ Posterior } & Incorrect & 54 & 7.51 & \multirow{2}{*}{$<0.0001^{*}$} \\
\cline { 2 - 4 } & Correct & 665 & 92.49 & \\
\hline
\end{tabular}

*Statistically significant value.

thus indicating perfect agreement between the sessions. Observer 1 values suggested an almost perfect agreement for the identification of defects on both surfaces (Table 2).

Table 3 shows the number of images that were correctly associated with the presence or absence of defects. There was strong evidence that the proportion of correct answers was significantly higher than the proportion of errors observed for the two surfaces.

Taking into account only the correct observations, Table 4 shows the conditions used in the analysis of variance and the parameters used to evaluate whether or not the observer responses were influenced by the application of the protocols. Strong significant evidence of the effect of the protocol used was detected only for the defects properly identified on the posterior surface. Therefore, we applied the
Table 4 - Analysis of variance results of the variables analyzed to determine the effects of the protocols used.

\begin{tabular}{c|c|c|c}
\hline Surface & $\begin{array}{c}\text { Response } \\
\text { distribution }\end{array}$ & $\begin{array}{c}\text { Statistical } \\
\text { adjustment }\end{array}$ & p-value \\
\hline Superior & Gaussian & -1071.35 & 0.6888 \\
\hline Posterior & Gaussian & -195.87 & $0.0003^{*}$ \\
\hline
\end{tabular}

*Statistically significant value.

Tukey test for multiple comparisons of means. The highest means were observed in Protocols 5 and 6, and a statistical difference was found only for those of Protocol 7, which had the lowest means (Table 5).

\section{Discussion}

In assessing the accuracy of defect identification, we found a significantly higher proportion of hits on both surfaces, as did Cara et al. ${ }^{14}$ and Utumi et $a l .{ }^{13}$ when employing MSCT to identify simulated cavitary defects. Cara et al. ${ }^{14}$ simulated defects, about $1 \mathrm{~mm}$ in diameter, in mandibular condyles, and the MSCT and single slice CT images that they obtained were evaluated in different reconstruction protocols, taking into account the orientation of the slices generated (axial slices $\times$ MPR images). The greatest sensitivity in identifying defects was found for MSCT MPRs. Later, Utumi et al. ${ }^{13}$ also evaluated defects, but, as already mentioned, they used three sizes of diamond burs. They compared the 
Table 5 - Comparison of mean hits on the posterior surface, in each of the nine protocols used.

\begin{tabular}{l|c|c|c|c}
\hline \multicolumn{1}{c|}{ Protocol } & Mean & $\begin{array}{c}\text { Standard } \\
\text { deviation }\end{array}$ & Median & $\begin{array}{c}\text { Tukey groups } \\
(\alpha=0.05)\end{array}$ \\
\hline 5 (0.6 mm + Sharpen Mild filter) & 0.97500 & 0.15711 & 1.0 & $\mathrm{~A}$ \\
\hline 6 (0.6 mm + S9 filter) & 0.97500 & 0.15711 & 1.0 & $\mathrm{~A}$ \\
\hline 9 (1.0 mm + S9 filter) & 0.95000 & 0.21932 & 1.0 & $\mathrm{AB}$ \\
\hline 4 (0.6 mm + none filter) & 0.95000 & 0.21932 & 1.0 & $\mathrm{AB}$ \\
\hline 8 (1.0 mm + Sharpen Mild filter) & 0.91250 & 0.28435 & 1.0 & $\mathrm{AB}$ \\
\hline 3 (0.2 mm + S9 filter) & 0.90000 & 0.30189 & 1.0 & $\mathrm{AB}$ \\
\hline 1 (0.2 mm + none filter) & 0.89873 & 0.30361 & 1.0 & $\mathrm{AB}$ \\
\hline 2 (0.2 mm + Sharpen Mild filter) & 0.88750 & 0.31797 & 1.0 & $\mathrm{AB}$ \\
\hline 7 (1.0 mm + none filter) & 0.87500 & 0.33281 & 1.0 & $\mathrm{~B} *$ \\
\hline
\end{tabular}

Protocols with same letters in the Tukey group row indicate that means do not differ. * Statistically significant value. traditional projections (coronal, axial and sagittal) with cross-sectional images, and obtained a sensitivity of $100 \%$ for identifying defects on the upper surface, using cross-sectional images.

Regarding studies comparing СBCT with other imaging modalities, we agree with the study results of Honda et al. ${ }^{7}$ and Honey et al. ${ }^{6}$ The reliability of diagnoses made by CBCT and MSCT in detecting erosions, osteophytes and sclerosis of the condyles was evaluated by Honda et al.,7 who found favorable results for CBCT when used for this purpose. Honey et al. ${ }^{6}$ observed CBCT bone defects on the lateral surface of dry condyles and compared the results with those obtained by other imaging modalities (panoramic radiography, conventional tomography and TMJ-specific panoramic projection). They found that CBCT accuracy was statistically higher than that of all other techniques. Only one study, by Hintze et al., ${ }^{15}$ found low values for CBCT sensitivity in identifying natural defects in dry mandibles (bone defects, flattening and osteophytes); however, since there was no statistical difference between CBCT and conventional tomography, it was concluded that CBCT was more indicated, due to the aforementioned advantages. In our study, cavitary defects were created to simulate erosion lesions, and the images were evaluated in nine reconstruction protocols, using various slice thicknesses and applying different filters. The higher proportion of images correctly associated with the presence or absence of defects corroborate the qualification of CBCT as a method of identifying these cavitary defects.

Some studies showed perfect intra-rater reliability for diagnosing cavitary lesions by CBCT, compared with other imaging modalities, such as corrected conventional tomography, ${ }^{15}$ conventional and TMJ-specific panoramic projections and conventional tomography. ${ }^{6}$ In our study, both intra- and inter-rater reliability yielded excellent results in identifying defects in the superior and posterior surfaces of the condyles.

Regarding the influence played by thickness, Perrela et al. ${ }^{8}$ compared MSCT images having $1.0 \mathrm{~mm}$ and $3.0 \mathrm{~mm}$ slice thickness (and the same interval between the slices), and observed that detection sensitivity and specificity of simulated defects in the body of the mandible were better in thinner slices. This is because some defects were extremely small and could go unnoticed in the $3.0 \mathrm{~mm}$ slices (partial volume effect). In the present research, the worst statistically significant result in identifying posterior surface defects was found in a protocol with the thickest slice used $(1.0 \mathrm{~mm})$, although all the defects were greater than the slice thickness used. On the other hand, it was expected that the best results would be found with thinner slices $(0.2 \mathrm{~mm})$; however, this was not the case. One possible explanation could be that, although very thin slices may increase diagnostic accuracy, they add considerable noise to the image and this may hinder the evaluation significantly. ${ }^{16}$

The role of the filters in diagnosing simulated 
external root resorption was studied by Vaz et al. ${ }^{12}$ The results for sensitivity, accuracy and negative predictive values were better for the enhancement filter (Sharpen $3 \times 3)$ than the smoothing filters $(S 9$, Smooth and Smooth $3 \times 3$ ), also used. One possible explanation given by the authors is that enhanced filters have good delineation of anatomic limits. In agreement with our results, the protocols using the enhancement filter (Sharpen Mild) presented the best results in identifying the defects on the posterior surface.

The post-processing possibilities of CBCT images may contribute significantly to the diagnostic capabilities of this exam. However, with no substantial clinical and scientific basis, the use of these resources may give rise to misinterpretation, and thus compromise the patient's treatment plan. Finally, attention should be given to the in vitro character of this and most of the studies mentioned, and even

\section{References}

1. Berni Neto RC, Freitas C, Gouveia AT, Fenyo-Pereira M, Bolzan M. [TMJ: study of the osseous abnormalities ocurrence by linear tomography]. Rev ABRO. 2003 Jul-Dec;4(2):81-4. Portuguese.

2. Alexiou KE, Stamatakis HC, Tsiklakis K. Evaluation of the severity of temporomandibular joint osteoarthritic changes related to age using cone beam computed tomography. Dentomaxillofac Radiol. 2009 Mar;38(3):141-7. DOI: 10.1259/ $\mathrm{dmfr} / 59263880$.

3. Larheim TA, Dale K, Tveito L. Radiographic abnormalities of the temporomandibular joint in children with juvenile rheumatoid arthritis. Acta Radiol Diagn (Stockh). 1981 May;22(3A):277-84.

4. Santos LAN, Campos PSF, Paula AMP, Martelli Júnior H, Melo Filho MR. [Image of juvenile idiopathic arthritis in mandibular condyle - case report]. Rev ABRO. 2005 JanJun;6(1):29-34. Portuguese.

5. Hussain AM, Packota G, Major PW, Flores-Mir C. Role of different imaging modalities in assessment of temporomandibular joint erosions and osteophytes: a systematic review. Dentomaxillofac Radiol. 2008 Feb;37(2):63-71. DOI: 10.1259/dmfr/16932758.

6. Honey OB, Scarfe WC, Hilgers MJ, Klueber K, Silveira AM, Haskell BS, et al. Accuracy of cone-beam computed tomography imaging of the temporomandibular joint: comparisons with panoramic radiology and linear tomography. Am J Orthod Dentofacial Orthop. 2007 Oct;132(4):429-38. more so to the fact that certain in vivo conditions cannot be perfectly reproduced in the laboratory. Thus, we suggest that the results of this research work should be applied under clinical conditions, thereby confirming and establishing the correspondence between in vitro and in vivo findings.

\section{Conclusions}

Based on the results of the current study, all protocols proposed have proved suitable for the identification of cavitary defects in the superior surface of the mandibular condyle. However, the protocol of a $1.0 \mathrm{~mm}$-thick slice and no filter proved to be the worst for identifying the defects on the posterior surface.

\section{Acknowledgements}

The authors wish to thank Professor Marcelo Alves, $\mathrm{PhD}$, for carrying out the statistical analysis.

7. Honda K, Larheim TA, Maruhashi K, Matsumoto K, Iwai K. Osseous abnormalities of the mandibular condyle: diagnostic reliability of cone beam computed tomography compared with helical computed tomography based on an autopsy material. Dentomaxillofac Radiol. 2006 May;35(3):152-7.

8. Perrella A, Borsatti MA,Tortamano IP, Rocha RG, Cavalcanti MG. Validation of computed tomography protocols for simulated mandibular lesions: a comparison study. Braz Oral Res. 2007 Apr-Jun;21(2):165-9.

9. Eickholz P, Riess T, Lenhard M, Hassfeld S, Staehle HJ. Digital radiography of interproximal bone loss; validity of different filters. J Clin Periodontol. 1999 May;26(5):294-300.

10. Haiter-Neto F, Pontual AA, Frydenberg M, Wenzel A. Detection of non-cavitated approximal caries lesions in digital images from seven solid-state receptors with particular focus on task-specific enhancement filters. An ex vivo study in human teeth. Clin Oral Investig. 2008 Sep;12(3):217-223. DOI: 10.1007/s00784-007-0173-5.

11. Wenzel A, Haiter-Neto F, Frydenberg M, Kirkevang LL. Variable-resolution cone-beam computerized tomography with enhancement filtration compared with intraoral photostimulable phosphor radiography in detection of transverse root fractures in an in vitro model. Oral Surg Oral Med Oral Pathol Oral Radiol Endod. 2009 Dec;108(6):939-45. DOI: 10.1016/j.tripleo.2009.07.041.

12. Vaz SLA, Vasconcelos TV, Neves FS, Freitas DQ, HaiterNeto F. Influence of cone-beam computed tomography enhancement filters on diagnosis of simulated external root 
resorption. J Endod. 2012 Mar;38(3):305-8. DOI: 10.1016/j. joen.2011.10.012.

13. Utumi ER, Perrella A, Albuquerque MAP, Adde CA, Rocha RG, Cavalcanti MGP. Evaluation of simulated bone lesion in the head of the mandible by using multislice computed tomography. J Appl Oral Sci. 2009 Sep-Oct;17(5):521-6.

14. Cara ACB, Gaia BF, Perrella A, Oliveira JXO, Lopes PML, Cavalcanti MGP. Validity of single- and multislice CT for assessment of mandibular condyle lesions. Dentomaxillofac Radiol. 2007 Jan;36(1):24-7.
15. Hintze $\mathrm{H}$, Wiese M, Wenzel A. Cone beam CT and conventional tomography for the detection of morphological temporomandibular joint changes. Dentomaxillofac Radiol. 2007 May;36(4):192-7.

16. Liang X, Jacobs R, Hassan B, Li L, Pauwels R, Corpas L, et al. A comparative evaluation of cone beam computed tomography (CBCT) and multi-slice CT (MSCT) Part I. On subjective image quality. Eur J Radiol. 2010 Aug;75(2):265-9. DOI: 10.1016/j. ejrad.2009.03.042. 